JAMA. 2018 November 27; 320(20): 2154. doi:10.1001/jama.2018.15946.

\title{
Baseline Genotype Testing to Assess Drug Resistance Before Beginning HIV Treatment-Reply
}

\author{
Michael S. Saag, MD' ${ }^{1}$, Huldrych F. Günthard, MD², and Davey M. Smith, MD \\ Jody W. Zylke, MD [Deputy Editor]
}

${ }^{1}$ School of Medicine, University of Alabama at Birmingham. ${ }^{2}$ University Hospital Zurich, Zurich, Switzerland. ${ }^{3}$ University of California, San Diego, La Jolla.

\section{In Reply}

As stated in the panel's report, ${ }^{1}$ the relative absence of resistance-conferring mutations for InSTI drugs supports the recommendation to not test routinely for InSTI resistance before start of HIV treatment. A summary of existing data regarding the relative lack of transmission of InSTI resistance- conferring mutations was published this year. ${ }^{2}$

As discussed by Dr Ambrosioni and colleagues, the relatively high prevalence of InSTI polymorphisms is of some concern. We agree that it is worth monitoring the effect of InSTI polymorphisms over time, especially as they may potentially affect the efficacy of 2-drug, InSTI-based therapy. To date, however, there are few data indicating that these mutations have meaningful clinical effect on virologic response, especially to InSTI agents recommended for initial therapy.

One of the main reasons the recommendations are updated every 2 years is to incorporate new data that emerge regarding treatment of patients with and at risk for HIV infection. Since relevant and important data often emerge quickly in this dynamic field, we are grateful to Ambrosioni and colleagues for putting this issue on the "watch list" for future recommendations.

\section{References}

1. Saag MS, Benson CA, Gandhi RT, et al. Antiretroviral drugs for treatment and prevention of HIV infection in adults: 2018 recommendations of the International Antiviral Society-USA panel. JAMA. 2018;320(4):379-396. doi:10.1001/jama.2018.8431 [PubMed: 30043070]

Corresponding Author: Michael S. Saag, MD, School of Medicine, University of Alabama at Birmingham, 845 19th St S, BBRB 256, Birmingham, AL 35294 (msaag@uabmc.edu).

Conflict of Interest Disclosures: The authors have completed and submitted the ICMJE Form for Disclosure of Potential Conflicts of Interest. Dr Saag reported receiving grants and personal fees from Merck, Gilead, and ViiV. Dr Günthard reported receiving grants from the National Institutes of Health, Swiss National Science Foundation, Swiss HIV Cohort Study, University of Zurich, Yvonne Jacob Foundation, Systems X, Gilead, and Roche and receiving personal fees from Merck, Gilead, Sandoz, and Teva. No other disclosures were reported. 
2. Günthard HF, Calvez V, Paredes R, et al. Human immunodeficiency virus drug resistance: 2018 recommendations of the International Antiviral Society-USA panel. Clin Infect Dis. 2018;67:1-11. [PubMed: 29340593] 